\title{
Role of taxanes in advanced prostate cancer
}

\author{
J. Cassinello ${ }^{1}$, J. Carballido Rodríguez ${ }^{2}$, L. Antón Aparicio ${ }^{3}$ \\ 1. Medical Oncology Service, Guadalajara University Hospital Guadalajara, Spain \\ 2. Urology Service, Puerta de Hierro University Hospital, Madrid, Spain \\ 3. Medical Oncology Service, CHU Hospital, La Coruña, Galicia, Spain
}

\begin{abstract}
Advanced prostate cancer is an androgen-dependent disease for which the initial treatment is an androgen deprivation maneuver. However, some primary resistances to hormonal treatment occur with increasing incidence throughout the evolution of the disease. The taxanes, docetaxel and cabazitaxel, exert their action at multiple levels at the tumor cell: besides inhibiting the mitosis and inducing the cell death, they induce the nuclear accumulation of FOXO1, a potent nuclear factor that acts against the activation of androgen receptor inhibiting the transcription of AR-V7 variant associated with the development of resistances to abiraterone and enzalutamide. Docetaxel, as first-line therapy, and cabazitaxel, as second-line therapy, have demonstrated to increase the survival in castration-resistant prostate cancer. The results from last studies either on high-risk localized disease or on androgen-sensitive tumors demonstrate the increasing role of taxanes at earlier states of prostate cancer.
\end{abstract}

\section{Keywords}

Prostate cancer; Docetaxel; Cabazitaxel; Androgen deprivation therapy; AR splice variant 


\section{Introduction}

Prostate cancer (PC) is the most prevalent urogenital malignancy and the second most common cause of cancer death in men in Europe. Age-standardized incidence and mortality rates per 100,000 population per year are 96.0 and 19.3 in Europe and 96.8 and 15.2 in Spain, respectively [1].

After radical local therapy, either with radical prostatectomy or radiotherapy, about $30 \%$ of patients develop advanced disease. Long-acting luteinizing hormone-releasing hormone (LHRH) agonists are currently the main form of androgen deprivation therapy (ADT). Complete or maximal androgen blockade ( $\mathrm{CAB}$ or $\mathrm{MAB})$ consists in the addition of an antiandrogen to a LHRH antagonist. Despite providing very good preliminary results, according to meta-analysis and systematic reviews, CAB appears to provide a small 5-year survival advantage of less than $5 \%$ compared to monotherapy with LHRH agonist [2, 3].

Taxanes are currently the only cytostatic class of drugs able to prolong survival in men with metastatic castration-resistant PC (mCRPC). Until recently, docetaxel was the only effective agent for the treatment of mCRPC. Now cabazitaxel, a new taxane, has demonstrated an improvement in overall survival (OS) and progression-free survival (PFS) due to its ability to overcome docetaxel resistance in vitro and in vivo. This review aims to summarize the current role of taxanes in castration-sensitive and castrationresistant advanced PC and their potential new active role in locally advanced and non-metastatic PC.

\section{Role of taxane agents in prostate cancer}

\section{General overview}

Taxane agents play an important role in PC disease. In high-risk patients with locally advanced disease, docetaxel has been tested before prostatectomy with promising results [4], showing activity in PC. They also show activity and prolong OS in advanced non-castration-resistant PC (non-CRPC) in combination with ADT (docetaxel, CHAARTED study [5]), as first-line treatment in castration-resistant PC (CRPC) patients (docetaxel, TAX-327 study [6]) and, finally, as second-line treatment in CRPC after progression to docetaxel (cabazitaxel, TROPIC study [7]).

Taxanes are mitotic inhibitors currently used as first- and second-line therapies for CRPC patients. Their mechanisms of action are multi-targeting (Fig. 1). They inhibit spindle disassembly by stabilizing the microtubule spindle, leading to cell cycle arrest and the disruption of many pathways, including ligand-dependent and ligand-independent androgen receptor (AR) nuclear translocation, which is mediated by microtubules [8]. Taxanes also induce the nuclear accumulation of FOXO1, a potent ARsuppressive nuclear factor that has been shown to inhibit the transcription of AR-V7 [8], a prevalent AR splice variant associated with enzalutamide and abiraterone resistance. In fact, detection of AR-V7 in circulating tumor cells from men with CRPC is not associated with primary resistance to taxanes. Therefore, AR-V7 may potentially serve as a treatment-selection marker in CRPC patients [9]. 

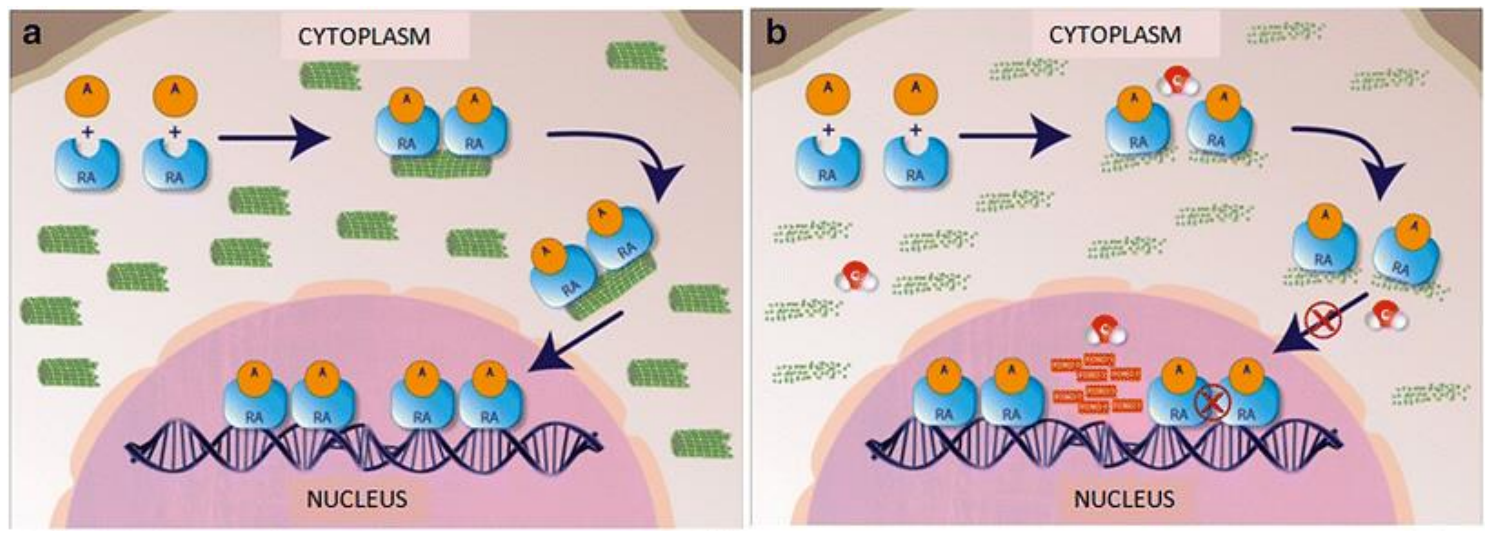

Fig. 1. Mode of action of cabazitaxel $(\mathrm{Cbz})$ : a normal tumoral cell; b $\mathrm{Cbz}$ action on the tumoral cell at three levels: 1 at microtubules as cytotoxic; 2 at RA impeding its translocation to nucleus; 3 at the nucleus, increasing the FOXO1 concentration

It is already known that PC is highly heterogeneous with the coexistence of AR-positive and ARnegative cancer cells in most patients, the latter being chemo-responsive. Targeting AR-positive cancer cells with endocrine therapies induces the clonal proliferation of AR-negative and AR-independent metastases [10]. There is growing evidence that differentiation to an AR-negative phenotype is a mechanism of resistance to AR pathway-targeted therapy leading to neuroendocrine differentiation [11]. Endocrine therapies can promote paradoxical tumor proliferation and lethal tumor phenotypes in patients with retinoblastoma loss or inactivation, a finding reported in $25-50 \%$ of cases of PC [12, 13].

Increased expression of N-cadherin in PC biopsies has been observed as early as 3 months after initiation of ADT and has been associated with loss of AR expression, epithelial-mesenchymal transition, cancer invasion and metastasis. Other mechanisms of resistance to AR-targeted agents include mutation, the generation of splice variants, and glucocorticoid receptor overexpression, which also possibly contribute to the induction of aggressive disease [14]. The specific role of taxanes in the different scenarios of advanced PC is reviewed here.

Taxanes (docetaxel) in combination with radiotherapy, ADT and zoledronic acid in high-risk locally advanced or metastatic PC

In locally advanced PC, docetaxel has shown activity in the RTOG 0521 study (in combination with radiotherapy) [15] and in the STAMPEDE study [16], both presented at 2015 ASCO congress. The STAMPEDE study [16] recruited men with high-risk locally advanced or metastatic PC starting hormone therapy. A total of 2962 patients were randomly allocated 2:1:1:1 to hormone therapy, hormone therapy plus docetaxel, hormone therapy plus zoledronic acid, and hormone therapy plus docetaxel plus zoledronic acid. In high-risk locally advanced patients, a statistically significant improvement in failurefree survival was observed from adding docetaxel to hormone therapy, but not in OS, may be due to the fact that the number of events is still very small. In the RTOG 0521 study [15] high-risk, localized PC patients benefited from adjuvant docetaxel which improved the OS from 89 to $93 \%$ at 4 years. Both studies reinforce the importance and the central role of taxanes-based chemotherapy in this disease at earlier states. 


\section{Taxanes (docetaxel) in combination with ADT in non-castration-resistant prostate cancer (non-CRPC)}

The combination of ADT and chemotherapy in advanced non-CRPC is under debate. Attacking the de novo testosterone-independent cells with chemotherapy early is an argument in favor of the use of this combination. An argument against this approach is the fact that some patients will never need chemotherapy, sparing the associated toxicity.

In the CHAARTED study (E3805) [5], the results from non-CRPC patients, who were randomized to receive ADT either continuously alone or combined with six cycles of docetaxel at standard dose, were presented at the 2014 ASCO meeting. A median OS of 44 months for ADT-treated patients was obtained, compared to 57.6 months for chemotherapy plus ADT-treated patients [HR $=0.61$ (95\% CI: 0.47-0.80); $p=0.003$ ]. This difference was even higher in high-volume metastatic patients (32.9 vs. 49.2 months; $\mathrm{HR}=0.6 ; p=0.006$ ). The authors concluded that six cycles of chemotherapy in addition to ADT represent an appropriate option for men with metastatic PC who are suitable for docetaxel therapy. Men with high-volume ADT-naive metastatic disease should be considered for ADT plus docetaxel based on the results of the E3805 CHAARTED study [17]. The STAMPEDE trial [16] included patients with newly diagnosed metastatic PC that was node positive, high-risk locally advanced or relapsing after previous radical local therapy. The patients were randomized to standard of care hormonal therapy plus docetaxel, zoledronic acid or both. Regarding M1 patients, the difference in OS between patients treated with ADT + docetaxel and the control arm was 22 months $(p=0.002)$. It is important to emphasize that in the STAMPEDE trial no differences were made between low and high-volume disease, raising the issue of whether should all patients receive docetaxel, irrespective of the volume or the risk of the disease, or not.

In a previous study, the GETUG-AFU 15 study [18], chemotherapy and hormone therapy combination prolonged disease-free survival (DFS) in comparison to ADT but did not show any survival advantage in non-CRPC patients. Indeed, this study was much smaller than E3805, and the number of patients in the ADT-only arm who received docetaxel for disease progression was $31 \%$ in the E3805 study and $62 \%$ in the GETUG-AFU 15 study. Interestingly, the patients in the ADT-only arm received docetaxel during post-progression treatment, raising the issue of the best timing of chemotherapy administration. Finally, GETUG-AFU 15 also had much fewer high-volume disease patients (22\% compared to $66.2 \%$ in E3805) and the number of cycles of docetaxel in the combination arm was higher than in the CHAARTED study.

In conclusion, the use of docetaxel should be considered in men with newly diagnosed metastatic or high-risk locally advanced disease if they are fit to receive chemotherapy.

\section{Taxanes (docetaxel) in symptomatic metastatic castration-resistant prostate cancer (mCRPC)}

CRPC must be defined before we proceed to define the role of docetaxel in this context. According to the European Association of Urology (EAU), an accurate definition of CRPC must meet the following criteria [19]: castrate serum testosterone levels, three consecutive increases in prostate-specific antigen (PSA), resulting in two $50 \%$ increases above the nadir, antiandrogen withdrawal for at least 4 weeks, PSA progression despite secondary hormonal manipulations and/or progression of bone (at least two new lesions upon bone scan) or soft tissue (RECIST criteria) lesions. Patients with mCRPC have a poor prognosis and a life expectancy of around 18-19 months.

M0 CRPC is characterized by the absence of visceral, skeletal, lymphatic and soft tissue metastasis detected by bone scan, computed tomography (CT) or magnetic resonance imaging (MRI), although the development of new and better imaging techniques (PET) will enable earlier detection of possible metastatic lesions. There is no treatment of choice for M0 CRPC patients, and their inclusion in clinical studies is encouraged. 
In M1 CRPC, two types of patients are defined: symptomatic or asymptomatic/mildly symptomatic. For symptomatic patients, chemotherapy is the treatment of choice; hormone therapy based on abiraterone (COU-AA-302 study) [20] or enzalutamide (PREVAIL study) [21] may be considered for those with few or no symptoms; however, docetaxel also seems to be active in asymptomatic or mildly symptomatic patients.

The first chemotherapy drug to demonstrate activity in CRPC a disease considered classically chemoresistant was mitoxantrone: in combination with prednisone, this drug showed a significant benefit in patients in terms of pain relief ( 29 vs. $12 \%, p=0.001)$ and duration of symptomatic response (43 vs. 18 weeks, $p<0.0001$ ), but had no impact on OS [22]. This combination of mitoxantrone and prednisone was the chemotherapy of choice for CRPC patients until 2004, when the results of two phase three studies demonstrated the superiority of docetaxel with prednisone over the mitoxantrone regimen. The most important of these two studies was the so-called TAX327 study, which randomized 1006 patients to receive either docetaxel $75 \mathrm{mg} / \mathrm{m}^{2}$ every 21 days, weekly docetaxel $30 \mathrm{mg} / \mathrm{m}^{2}$ or mitoxantrone $12 \mathrm{mg} / \mathrm{m}^{2}$ every 21 days; in all three arms, prednisone $5 \mathrm{mg}$ was also administered every $12 \mathrm{~h}$. The group of patients who received tri-weekly docetaxel had their risk of death reduced by $24 \%$ compared to the mitoxantrone arm, with a median survival rate of 18.9 and 16.5 months, respectively (HR $=0.76, p=0.009)$; pain was also significantly improved in the group receiving docetaxel every 3 weeks ( 35 vs. $22 \%, p=0.01$ ) as was quality of life ( 22 vs. $13 \%, p=0.009)$ compared to the patients receiving mitoxantrone [6].

Therefore, the regimen containing docetaxel $75 \mathrm{mg} / \mathrm{m}^{2}$ every 3 weeks in combination with prednisone $5 \mathrm{mg}$ every $12 \mathrm{~h}$ became the treatment of choice for patients with symptomatic CRPC. These favorable tri-weekly docetaxel data were confirmed in an update in 2008, with a median survival 19.2 vs. 16.3 months $(\mathrm{HR}=0.79, p=0.004)$ and a $5 \%$ increase in survival at 3 years $(18.6 \mathrm{vs} .13 .5 \%)$ [23]. In the subgroup analysis, we found that patients with visceral involvement and symptomatic patients with a Karnofsky score of less than $90 \%$ or with elevated PSA levels (greater than $114 \mathrm{ng} / \mathrm{ml}$ ) had worse outcomes. Specifically, it should be noted that symptomatic patients had a median survival rate of 17.1 vs 25.9 months for those with minimal symptoms. Interestingly, docetaxel is also superior to mitoxantrone in these symptomatic patients.

Regarding PSA response, it is interesting to note that, in the majority of patients, this was associated with an improvement in quality of life and alleviation of pain; in turn, the combination of biochemical response and decreased pain was associated with a significant improvement in survival [23]. Moreover, for patients unlikely to tolerate this 3-weekly regimen due to the presence of comorbidities, $50 \mathrm{mg} / \mathrm{m}^{2}$ docetaxel administered every 2 weeks could be a useful option [24].

\section{Taxanes (docetaxel) in asymptomatic or mildly symptomatic patients with CRPC}

Besides the presence of symptoms, there are no well-defined predictive factors to determine which asymptomatic or mildly symptomatic patients would benefit from docetaxel-based chemotherapy over hormonal maneuvers, including abiraterone or enzalutamide. There are general factors that predict rapidly progressive disease and shorter survival and justify the choice of docetaxel as first-line treatment: the presence of anemia, multiple metastatic sites, elevated LDH and alkaline phosphatase levels and specifically PSA doubling time (PSADT) of less than 55 days, in accordance with the international consensus.

Mildly symptomatic patients in whom chemotherapy may be preferred are those asymptomatic patients included in the COU-AA-302 study treated with abiraterone [20] and having a poor outcome. Docetaxel-based chemotherapy may also be considered in patients with anemia, elevated LDH levels, an elevated PSA doubling time value or the presence of lymph nodes plus visceral disease, as patients with visceral metastases were excluded from the COU-AA-302 study. 


\section{Taxanes (cabazitaxel) after docetaxel progression}

Four options with category 1 recommendations are now available after progression to docetaxel, including a cytostatic agent (cabazitaxel), two hormonal treatments (abiraterone and enzalutamide) and a radio-transmitter alpha particle (radium-223). There is no drug of choice because none of these treatments have been compared to one another, and the selection of therapy is currently based primarily on clinical characteristics such as previous therapy, the presence of visceral metastases or disease symptoms, adverse event profiles, life expectancy, comorbidities, performance status, cost and patient preference. A fifth option is retreatment with docetaxel in patients who have previously responded to this taxane; however, evidence supporting this option is not robust (phase 2 retrospective studies with a small number of patients) and has not, in any case, been shown to increase survival [25].

Cabazitaxel is a new generation taxane specifically designed to overcome docetaxel-generated resistance. Interestingly, this agent easily crosses the blood-brain barrier. Cabazitaxel was compared to mitoxantrone following docetaxel progression in the randomized, multicentre, international phase 3 TROPIC study [7]; this study compared cabazitaxel at a dose of $25 \mathrm{mg} / \mathrm{m}^{2}$ and mitoxantrone at a dose of $12 \mathrm{mg} / \mathrm{m}^{2}$ every 3 weeks; this was combined with $10 \mathrm{mg}$ of prednisone daily in both arms. Seven hundred and fifty-five ECOG 0-2 patients with CRPC, who had progressed during or after at least three cycles of docetaxel, were randomized to receive cabazitaxel $(n=378)$ or mitoxantrone $(n=377)$ until disease progression or unacceptable toxicity or for a maximum of ten cycles. Stratification was performed by ECOG 0-1 vs. 2 and measurable vs. non-measurable disease. The primary endpoint was OS; secondary endpoints included PFS, defined as the time between the date of randomization and PSA progression, tumor progression, increased symptoms or death.

The median survival of patients in the cabazitaxel arm was 15.1 months, whereas that in the mitoxantrone arm was 12.7 months. This difference translates into a reduction in the risk of death of $30 \%$ for patients receiving cabazitaxel $(\mathrm{HR}=0.70,95 \% \mathrm{CI}: 0.95-0.83, p<0.0001)$; median PFS was 2.8 months for cabazitaxel (95\% CI: 2.4-3.0) and 1.4 months for mitoxantrone (95\% CI: 1.4-1.7). With a median follow-up of 25.5 months, data confirmed that the 2-year survival rate was higher in patients treated with cabazitaxel [26]. Cabazitaxel also prolonged survival in comparison to mitoxantrone independently of the number of poor prognostic factors, including visceral disease [27]. Interestingly, the absolute benefit of cabazitaxel in the TROPIC study is of the same magnitude of docetaxel in the TAX 327 study, but the population was older, had received more treatment, and had received a taxane previously [26]. These results show that treatment with cabazitaxel significantly improves survival in patients with aggressive disease compared to mitoxantrone-prednisone (MP), including patients with visceral metastases.

The most common toxicities with cabazitaxel were neutropenia (primary prophylaxis was not allowed) and diarrhea; $28 \%$ of patients treated with cabazitaxel had febrile neutropenia, so it is considered advisable to assess the prophylactic use of hematopoietic growth factors under the following circumstances: patients aged more than 65 years old, which had been treated with radiotherapy or present with extensive bone disease or had prior history of febrile neutropenia with docetaxel. It is important to point out that it is safe for elderly patients to receive cabazitaxel taking into account that they are fit for chemotherapy [28]. In this regard, there is an ongoing study that focuses on the tolerability and efficacy of weekly cabazitaxel in unfit CRPC patients who progressed to docetaxel [29]. It is important to emphasize, as has already been mentioned, that, in relation to the cabazitaxel hematological toxicity which occurred in the TROPIC study, the use of primary prophylaxis with colony-stimulating factors was not permitted; however, with its more frequent use in daily clinical practice, the incidence of neutropenia has decreased notably [30].

The decision to start treatment with cabazitaxel after docetaxel progression should be based on individual patient characteristics in the absence of formal comparison to the alternative of hormonal treatment, either with abiraterone or enzalutamide. Refractoriness or early progression to docetaxel could be one argument for the indication of cabazitaxel, among others, as will be discussed later. 
Phase 3 studies underway with cabazitaxel in CRPC

The PROSELICA study (NCT01308580) comparing the administration of cabazitaxel at doses of 20 vs. $25 \mathrm{mg} / \mathrm{m}^{2}$ in CRPC patients who progressed to docetaxel is ongoing. The main endpoint is OS and the secondary endpoints are: safety, PFS, progression by PSA and tumor size, as well as pain or quality of life responses.

Another study is testing the value of front-line cabazitaxel in a phase 3 study called FIRSTANA (NCT01308567), which is comparing cabazitaxel tri-weekly at a dose of $25 \mathrm{vs.} 20 \mathrm{mg} / \mathrm{m}^{2}$ with a standard docetaxel $75 \mathrm{mg} / \mathrm{m}^{2}$ regimen every 3 weeks. In all three arms, prednisone $10 \mathrm{mg}$ daily is also administered. The primary endpoint is OS and secondary endpoints are PFS, radiographic PFS, tumor response in measurable disease, PSA responses, PFS biochemistry, pain control, time to development of skeletal complications and quality of life.

The third phase 3 study which has been launched concerns cabazitaxel in combination with prednisone with or without the anti-clusterin agent custirsen (NCT015788655) as second-line therapy, which aims to explore the activity and safety of cabazitaxel in combination.

\section{Profile of candidate patients for cabazitaxel therapy}

An analysis of the characteristics of the patients recruited into the TROPIC study [7], which could guide us in selecting an appropriate profile for cabazitaxel patients, revealed that $25 \%$ had visceral metastases, $92.6 \%$ had a performance status of ECOG $0-1,30 \%$ had undergone two or more prior chemotherapy regimens, including docetaxel at different dose levels, and more than $70 \%$ were refractory to docetaxel (progression during or within 3 months of docetaxel completion).

Basically, the type of patients to be treated with cabazitaxel would include those with mCRPC and suspected hormonal independence, especially those with more aggressive potential (Gleason > 7), rapid progression to mCRPC with primary ADT, and those who are clearly refractory to docetaxel, particularly if progression has occurred during treatment with this drug. Patients aged $\geq 70$ years showed similar general and hematological toxicity compared to patients below 70 years of age. Prior treatment with ketoconazole and baseline serum adrenal androgen levels may also have to be taken into account. However, this should not exclude patients with less aggressive clinical disease who, as we have seen, also benefit from treatment with cabazitaxel.

Finally, we cannot forget that, of all four therapeutic options after docetaxel progression, the cabazitaxel option requires the patient to have a good ECOG score to be used safely. This means that cabazitaxel must be used in the setting of the disease in which the patient maintains a performance status sufficient to receive a potentially toxic chemotherapy agent.

\section{Taxanes (cabazitaxel) as part of the sequential treatment in CRPC after docetaxel progression}

As already mentioned, in addition to cabazitaxel, there are a range of options available after progression to docetaxel, including two hormonal treatments (abiraterone and enzalutamide) and a radiotransmitter alpha particle (radium-223). All of these treatments are valid options, taking into account the characteristics of the patients and the drug availability. As we know, there is no drug of choice because none of these treatments have been compared head-to-head with one another. It is not yet known how great the incremental benefit obtained through the sequential use of the novel treatments will be. As a consequence, no specific sequence of post-docetaxel treatment can be recommended. Interestingly, as is the case with other common advanced tumors, the more drugs used, the better the outcome: using cabazitaxel and abiraterone-enzalutamide after progression to docetaxel, whatever the sequence of administration is used, is associated with longer survival than the use of just two of these drugs [31]. 


\section{Sequence of treatment after docetaxel progression: cabazitaxel and new hormonal therapies}

In a retrospective study of 113 patients progressing after docetaxel treatment, a better outcome was obtained by more patients receiving the cabazitaxel-abiraterone sequence than those receiving the abiraterone-cabazitaxel sequence; this included a lower risk of mortality [32].

In another retrospective study of CRPC patients having progressed after first-line treatment with docetaxel, 63 patients received cabazitaxel and then abiraterone and 69 patients received abiraterone and then cabazitaxel. Median OS was 19.1 and 17.0 months in the cabazitaxel-abiraterone sequence and abiraterone-cabazitaxel sequence, respectively $(p=0.36)$; median PFS and biochemical PFS were significantly longer in the cabazitaxel-abiraterone sequence vs. the opposite: 8.1 vs. 6.5 months $(p=0.050)$ and 9.5 vs. 7.7 months $(p=0.024)$, respectively. Minor differences in toxicity were observed based on therapy sequence; generally, toxicity from cabazitaxel could be severe, while abiraterone toxicity was milder. The authors concluded that patient survival did not differ significantly based on treatment sequence and that primary progression on cabazitaxel or abiraterone did not preclude a response to the other agent in CRPC patients. However, tumor response to both agents, particularly cabazitaxel, was lower when administered as later-line therapy in the selected study population [33].

Finally, Maines published data from 13 retrospective trials with 1016 patients, that included the following sequences in post-docetaxel setting: new hormonal therapy (NHA) $\rightarrow$ NHA (469 patients); NHA $\rightarrow$ cabazitaxel (318 patients); and cabazitaxel $\rightarrow$ NHA (229 patients). The 12-month OS rates in the three sequence groups were 28.5, 61.3, and 76.4\%, respectively. Data concerning major prognostic factors (median age, PS ECOG $0-1$ and $>2$, Gleason score $>8$ and the rate of bone, lymph nodes and visceral metastases) in the three groups were no substantially different between them. Despite the retrospective nature of the data, possible survival advantage could be observed when Cabazitaxel-based sequence is used [34].

\section{Are taxanes still active after abiraterone or enzalutamide?}

There are limited clinical and pre-clinical data to suggest the existence of cross-resistance between docetaxel and enzalutamide or abiraterone. In vitro studies indicate that taxanes may act by disrupting AR signaling. This may represent a potential mechanism for cross-resistance in mCRPC among taxanes and new hormonal agents [8]. However, taxanes also induce cell death by means of AR-independent mechanisms that may overcome prior hormone-therapy resistance and act against AR-negative cells.

\section{Docetaxel}

The efficacy of docetaxel remains controversial after abiraterone use according to various retrospective studies. In a retrospective study of docetaxel efficacy, in 35 out of $54 \mathrm{mCRPC}$ patients previously treated with abiraterone [35], no responses to docetaxel were seen in patients who did not attain a PSA decline $\geq 50 \%$ with abiraterone; docetaxel only resulted in $26 \%$ PSA response rate, a median time to PSA progression of 4.6 months and median OS of 12.5 months. In this study, the eight abiraterone-refractory patients were also refractory to docetaxel.

A more recent study [36] had the same results, indicating a cross-reaction between docetaxel and abiraterone; however, PSA responses were reported with docetaxel in patients who did not achieve a PSA decline $\geq 50 \%$ with abiraterone. In a third study [37], the outcomes of 86 mCRPC patients treated with docetaxel after abiraterone were studied; the authors found that confirmed PSA response rates, median PFS and median OS with docetaxel did not significantly differ between patients who experienced no PSA decline, $<50 \%$ decline and $\geq 50 \%$ decline with abiraterone. In the cohort of 54 patients with abirateronerefractory disease, the confirmed PSA response rate was $37 \%$. These data are comparable to the findings of Schweizer et al. [36], who observed PSA responses in $39 \%$ (7/18) of abiraterone-refractory patients. 
In summary, in the first study, no responses to docetaxel were seen in patients who did not attain a PSA decline $\geq 50 \%$ with abiraterone [35]; in the second study [36], PSA responses to docetaxel were reported in patients who did not achieve a PSA decline $\geq 50 \%$ with abiraterone; and, finally, in the third study, [37] PSA response rates, median PFS and median OS with docetaxel did not significantly differ between patients who experienced no PSA decline, $<50 \%$ decline and $\geq 50 \%$ decline with abiraterone.

Globally, we found that docetaxel efficacy in mCRPC patients was not clearly linked to the biochemical response to previous abiraterone therapy. In the absence of prospective studies, our data suggest that biochemical response to prior abiraterone therapy should not be used to guide patient selection for subsequent treatment with docetaxel.

\section{Cabazitaxel}

The influence of AR-targeted agents on the activity of cabazitaxel, a next-generation taxane developed to overcome docetaxel resistance, remains controversial.

In one study, the activity of cabazitaxel in CRPC after docetaxel and the novel endocrine agents, abiraterone and enzalutamide, was measured; 59 patients were treated with cabazitaxel, 37 of whom had previously received abiraterone and 9 of whom had previously received enzalutamide. These patients received a median of 7 months of abiraterone and 11 months of enzalutamide. A median of six cabazitaxel cycles were delivered with $\geq 50 \%$ PSA declines in $39 \%$, soft tissue radiological responses in $14 \%$ and symptomatic benefit in $24 \%$. Median OS and PFS were 15.8 and 4.6 months, respectively. The authors concluded that cabazitaxel showed a significant activity in patients who had already been treated with docetaxel, abiraterone and enzalutamide [38].

Another group reviews their data relating to 132 consecutive patients treated with cabazitaxel after docetaxel progression; they found that patients receiving AR-targeted agents after cabazitaxel demonstrated a twofold improvement in radiological and clinical progression and OS compared to those receiving AR-targeted agents before cabazitaxel, despite similar PSA response rates [39].

In a similar study, 79 patients who had progressed after docetaxel and abiraterone received cabazitaxel $25 \mathrm{mg} / \mathrm{m}^{2}$ every 3 weeks. A PSA decline $\geq 30 \%$ was achieved in 48 patients (62\%; $95 \%$ CI: 51-73) and a PSA decline $>50 \%$ was achieved in $35 \%$ (95\% CI: 25-47). The authors concluded that cabazitaxel and AR pathway inhibitors are not cross-resistant due to the fact that cabazitaxel does not act mainly through AR axis inhibition [40].

Taken together, these data suggest that cabazitaxel is an effective taxane in patients with mCRPC, as well as in pre-clinical models that are resistant to AR-targeted agents. As a consequence, we can conclude that there is a lack of cross-resistance between cabazitaxel and AR-targeted agents, because cabazitaxel acts mainly through AR-independent mechanisms. In an enzalutamide-resistant xenograft model, cabazitaxel was associated with a similar PSA response but greater anti-tumor activity and prolonged PFS when compared to docetaxel [41]. We should feel reassured by the existence of an active agent that is able to prolong survival in patients progressing on new AR-targeted agents.

In the current treatment landscape, with healthcare expenditure increasing exponentially, we should stop thinking that one specific AR-targeted agent is good for all the patients because it is easy to use and well tolerated. Some patients do not respond to these drugs and stimulation of the clonal component of the disease might render the tumor even more aggressive and reduce the patient's chances of survival.

At last, prospective randomized studies are needed to unambiguously confirm the existence of crossresistance between AR-targeted agents and determine which sequence of therapies would be most beneficial to men with mCRPC. 
It can be concluded that the true impact of sequential therapy is not yet established; however, based on limited clinical data, sequential treatment with cabazitaxel, abiraterone and enzalutamide has shown clinical activity and should be considered in patients with mCRPC [32].

\section{Conclusions}

In recent years, the therapeutic scenario of advanced PC has changed dramatically. Taxanes play a central role in this new era of PC treatment. Besides docetaxel, we can use now cabazitaxel as second-line treatment of $\mathrm{mCRPC}$, with studies concerning its use as first-line treatment ongoing. Moreover, the role of taxanes has been reinforced with the presentation in the 2014 ASCO congress of the CHAARTED study results, which demonstrate the importance of docetaxel in combination with ADT in patients with non-CRPC. Moreover, two recent studies presented at the 2015 ASCO congress - the STAMPEDE and the RTOG 0521 studies - have reinforced the value of taxane-based chemotherapy at the earlier states of the PC. The ultimate goal of all cancer therapies is to prolong patient survival and improve quality of life. In patients with CRPC, docetaxel and cabazitaxel achieve these two objectives. Actually, the current SEOM (Sociedad Española de Oncología Médica) [42] and ESMO (European Society of Medical Oncology) [43] clinical guidelines reflect the importance of taxanes in prostate cancer treatment. We can conclude that taxanes (docetaxel and cabazitaxel) are essential agents in the management of advanced PC.

\section{Acknowledgments}

Medical writing assistance was provided by PIVOTAL S.L, funded by Sanofi. The authors are fully responsible for all content and editorial decisions for this manuscript. The authors indicate that they do not have any conflict of interests to declare.

\section{Compliance with ethical standards}

\section{Informed consent}

This article is a review of published studies, thus achievement of the patient Informed consent was not necessary.

\section{Research involving human participants and/or animals}

This article does not contain any studies with human participants or animals performed by any of the authors.

\section{References}

1. Ferlay J, Soerjomataram I, Ervik M, Dikshit R, Eser S, Mathers C, et al. GLOBOCAN 2012 v1.0, cancer incidence and mortality worldwide: IARC CancerBase No. 11. http://globocan.iarc.fr. 2013.

2. Prostate Cancer Trialists' Collaborative Group. Maximum androgen blockade in advanced prostate cancer: an overview of the randomised trials. Lancet. 2000;355(9214):1491-8.

3. Seidenfeld J, Samson DJ, Hasselblad V, Aronson N, Albertsen PC, Bennett CL, et al. Singletherapy androgen suppression in men with advanced prostate cancer: a systematic review and meta-analysis. Ann Intern Med. 2000;132(7):566-77.

4. Chi KN, Chin JL, Winquist E, Klotz L, Saad F, Gleave ME. Multicenter phase II study of combined neoadjuvant docetaxel and hormone therapy before radical prostatectomy for patients with high risk localized prostate cancer. J Urol. 2008;180(2):565-70. 
5. Sweeney C, Chen Y, Carducci MA, Liu G, Jarrard FD, Eisenberger MA, et al. Impact on overall survival with chemohormonal therapy versus hormonal therapy for hormone-sensitive newly metastatic prostate cancer: an ECOG-led phase III randomized trial. 50th annual meeting of American Society of Clinical Oncology (June 2014). Abstract LBA2. 2015.

6. Tannock IF, de WR, Berry WR, Horti J, Pluzanska A, Chi KN, et al. Docetaxel plus prednisone or mitoxantrone plus prednisone for advanced prostate cancer. N Engl J Med. 2004;351(15):1502-12.

7. de Bono JS, Oudard S, Ozguroglu M, Hansen S, Machiels JP, Kocak I, et al. Prednisone plus cabazitaxel or mitoxantrone for metastatic castration-resistant prostate cancer progressing after docetaxel treatment: a randomised open-label trial. Lancet. 2010;376(9747):1147-54.

8. Fitzpatrick JM, de Wit R. Taxane mechanisms of action: potential implications for treatment sequencing in metastatic castration-resistant prostate cancer. Eur Urol. 2014;65(6):1198-204

9. Antonarakis ES, Lu C, Luber B, Wang H, Chen Y, Nakazawa M, et al. Androgen receptor splice variant 7 and efficacy of taxane chemotherapy in patients with metastatic castrationresistant prostate cancer. JAMA Oncol. 2015;1(5):582-91.

10. Beltran H, Rickman DS, Park K, Chae SS, Sboner A, MacDonald TY, et al. Molecular characterization of neuroendocrine prostate cancer and identification of new drug targets. Cancer Discov. 2011;1(6):487-95.

11. Tagawa ST. Neuroendocrine prostate cancer after hormonal therapy: knowing is half the battle. J Clin Oncol. 2014;32(30):3360-4.

12. Pezaro CJ, Omlin A, Lorente D, Nava RD, Ferraldeschi R, Bianchini D, et al. Visceral disease in castration-resistant prostate cancer. Eur Urol. 2014;65(2):270-3.

13. Zong Y, Goldstein AS. Adaptation or selection-mechanisms of castration-resistant prostate cancer. Nat Rev Urol. 2013;10(2):90-8.

14. Arora VK, Schenkein E, Murali R, Subudhi SK, Wongvipat J, Balbas MD, et al. Glucocorticoid receptor confers resistance to antiandrogens by bypassing androgen receptor blockade. Cell. 2013;155(6):1309-22.

15. Sandler HM, Hu C, Rosenthal SA, Sartor O, Gomella LG, Amin M, et al. A phase III protocol of androgen suppression (AS) and 3DCRT/IMRT versus AS and 3DCRT/IMRT followed by chemotherapy (CT) with docetaxel and prednisone for localized, high-risk prostate cancer (RTOG 0521). J Clin Oncol. 2015;33 (suppl; abstr LBA5002).

16. James ND, Sydes MR, Mason MD, Clarke NW, Dearnaley DP, Spears MR, et al. Docetaxel and/or zoledronic acid for hormone-naïve prostate cancer: first overall survival results from STAMPEDE (NCT00268476). J Clin Oncol. 2015;33 (suppl; abstr 5001).

17. NCCN. Prostate cancer update. NCCN guidelines version 1. 2015.

18. Gravis G, Fizazi K, Joly F, Oudard S, Priou F, Esterni B, et al. Androgen-deprivation therapy alone or with docetaxel in non-castrate metastatic prostate cancer (GETUG-AFU 15): a randomised, open-label, phase 3 trial. Lancet Oncol. 2013;14(2):149-58.

19. Heidenreich A, Bastian PJ, Bellmunt J, Bolla M, Joniau S, Mason MD, et al. European association of urology: guidelines of prostate cancer 2012.

20. Ryan CJ, Smith MR, de Bono JS, Molina A, Logothetis CJ, de SP, et al. Abiraterone in metastatic prostate cancer without previous chemotherapy. N Engl J Med. 2013;368(2):138-48.

21. Beer TM, Armstrong AJ, Rathkopf DE, Loriot Y, Sternberg CN, Higano CS, et al. Enzalutamide in metastatic prostate cancer before chemotherapy. N Engl J Med. 2014;371(5):424-33.

22. Tannock IF, Osoba D, Stockler MR, Ernst DS, Neville AJ, Moore MJ, et al. Chemotherapy with mitoxantrone plus prednisone or prednisone alone for symptomatic hormone-resistant prostate cancer: a Canadian randomized trial with palliative end points. J Clin Oncol. 1996;14(6):175664.

23. Berthold, Pond GR, Soban F, de WR, Eisenberger M, Tannock IF. Docetaxel plus prednisone or mitoxantrone plus prednisone for advanced prostate cancer: updated survival in the TAX 327 study. J Clin Oncol. 2008;26(2):242-5.

24. Kellokumpu-Lehtinen PL, Harmenberg U, Joensuu T, McDermott R, Hervonen P, Ginman C, et al. 2-Weekly versus 3 -weekly docetaxel to treat castration-resistant advanced prostate cancer: a randomised, phase 3 trial. Lancet Oncol. 2013;14(2):117-24.

25. Oudard S, Kramer G, Creppy L, Loriot Y, Steinbjoern H, Holmberg M, et al. Management of metastatic castration-resistant prostate cáncer (mCRPC) after an initial good response to firstline docetaxel: a retrospective study on 270 patients. Eur J Cancer. 2011;47(Suppl 1):abstract 7049.

26. Bahl A, Oudard S, Tombal B, Ozguroglu M, Hansen S, Kocak I, et al. Impact of cabazitaxel on 2-year survival and palliation of tumour-related pain in men with metastatic castration-resistant prostate cancer treated in the TROPIC trial. Ann Oncol. 2013;24(9):2402-8. 
27. Chi KN, de Bono JS, Bahl A, Oudard S, Tombal B, Ozguroglu M, et al. Analysis of overall survival for patients with different prognostic risk factors treated with cabazitaxel and prednisone after docetaxel in the TROPIC trial. Ann Oncol. 2014;25(Suppl 4):iv255-79 (Poster 771P).

28. Heidenreich A, Bracarda S, Mason M, Ozen H, Sengelov L, Van OI, et al. Safety of cabazitaxel in senior adults with metastatic castration-resistant prostate cancer: results of the European compassionate-use programme. Eur J Cancer. 2014;50(6):1090-9.

29. Perez Valderrama B, Mellado B, Juan MJ, Fernandez O, Fernandez-Parra E, Ochoa de Olza M, et al. Preliminary results of a phase II study of weekly cabazitaxel in "unfit" metastatic castration resistant prostate cancer (mCRPC) patients after docetaxel treatment (SOGUGCABASEM trial). Ann Oncol. 2014;25(suppl 4):iv270 (783P).

30. Castellano D, Anton Aparicio LM, Esteban E, Sanchez-Hernandez A, Germa JR, Batista N, et al. Cabazitaxel for metastatic castration-resistant prostate cancer: safety data from the Spanish expanded access program. Expert Opin Drug Saf. 2014;13(9):1165-73.

31. Oudard S, Angelergues A, Gonzalez Maeso I, Delanoy N, Flechon A, Ozguroglu M, et al. Prognostic factors for survival and sequencing of life extending therapies in mCRPC patients in post-docetaxel setting. Ann Oncol. 2014;25(suppl 4):iv255-79 (789 P).

32. Sonpavde G, Bhor M, Hennessy D, Bhowmik D, Shen L, Nicacio L, et al. Sequencing of cabazitaxel and abiraterone acetate after docetaxel in metastatic castration-resistant prostate cancer: treatment patterns and clinical outcomes in multicenter community-based US oncology practices. Clin Genitourin Cancer. 2015;13(4):309-18.

33. Wissing MD, Coenen JL, van den BP, Westgeest HM, van den Eertwegh AJ, Van OI, et al. CAST: a retrospective analysis of cabazitaxel and abiraterone acetate sequential treatment in patients with metastatic castrate-resistant prostate cancer previously treated with docetaxel. Int J Cancer. 2015;136(6):E760-72.

34. Maines F, Caffo O, Veccia A, Trentin C, Tortora G, Galligioni E, et al. Sequencing new agents after docetaxel in patients with metastatic castration-resistant prostate cancer. Crit Rev Oncol Hematol. 2015;96(3):498-506.

35. Mezynski J, Pezaro C, Bianchini D, Zivi A, Sandhu S, Thompson E, et al. Antitumour activity of docetaxel following treatment with the CYP17A1 inhibitor abiraterone: clinical evidence for cross-resistance? Ann Oncol. 2012;23(11):2943-7.

36. Schweizer MT, Zhou XC, Wang H, Bassi S, Carducci MA, Eisenberger MA, et al. The influence of prior abiraterone treatment on the clinical activity of docetaxel in men with metastatic castration-resistant prostate cancer. Eur Urol. 2014;66(4):646-52.

37. Azad AA, Leibowitz-Amit R, Eigl BJ, Lester R, Wells JC, Murray RN, et al. A retrospective, Canadian multi-center study examining the impact of prior response to abiraterone acetate on efficacy of docetaxel in metastatic castration-resistant prostate cancer. Prostate. 2014;74(15):1544-50.

38. Pezaro CJ, Omlin AG, Altavilla A, Lorente D, Ferraldeschi R, Bianchini D, et al. Activity of cabazitaxel in castration-resistant prostate cancer progressing after docetaxel and nextgeneration endocrine agents. Eur Urol. 2014;66(3):459-65.

39. Aggarwal R, Harris A, Formaker C, Small EJ, Molina A, Griffin TW, et al. Response to subsequent docetaxel in a patient cohort with metastatic castration-resistant prostate cancer after abiraterone acetate treatment. Clin Genitourin Cancer. 2014;12(5):e167-72.

40. Al Nakouzi N, Le MS, Albiges L, Wang C, Beuzeboc P, Gross-Goupil M, et al. Cabazitaxel remains active in patients progressing after docetaxel followed by novel androgen receptor pathway targeted therapies. Eur Urol. 2015;68(2):228-35.

41. Scher HI, Beer TM, Higano CS, Anand A, Taplin ME, Efstathiou E, et al. Antitumour activity of MDV3100 in castration-resistant prostate cancer: a phase 1-2 study. Lancet. 2010;375(9724):1437-46.

42. Cassinello J, Climent MA, Del Gonzalez AA, Mellado B, Virizuela JA. SEOM clinical guidelines for the treatment of metastatic prostate cancer. Clin Transl Oncol. 2014;16(12):1060-6.

43. Parker C, Gillessen S, Heidenreich A, Horwich A. Cancer of the prostate: ESMO clinical practice guidelines for diagnosis, treatment and follow-up. Ann Oncol. 2015;26(Suppl 5):v6977. 